\title{
Ausschreibung für den Wettbewerb um die Carl-Diem-Plakette 1999/2000
}

1. Der Wettbewerb um die CARL-DIEM-Plakette dient vor allem der Förderung des sportwissenschaftlichen Nachwuchses. Zu diesem Zweck verleiht der DEUTSCHE SPORTBUND alle zwei Jahre für eine hervorragende sportwissenschaftliche Arbeit in deutscher Sprache die CARL-DIEM-Plakette. Diese wird in den zwei Sektionen Naturwissenschaft/ Medizin und Geistes-/Sozialwissenschaften vergeben.

2. Wichtige Kriterien für die Beurteilung der eingereichten Arbeiten sind ihr wissenschaftlicher Charakter, die neuen Erkenntnisse der vorgelegten Untersuchung sowie ihre Originalität und Aktualität.

3. Mit der Verleihung der CARL-DiEM-Plakette (Erster Preis) ist ein Geldpreis bis zur Höhe von DM 5000,- verbunden. Neben dem Ersten Preis können auch Zweite Preise vergeben und Lobende Anerkennungen ausgesprochen werden, die ebenfalls mit Geldpreisen dotiert sind. Die Gesamtpreissumme beträgt bis zu DM 25000,-.

4. Die Preisträger werden in einer Festakademie geehrt, die gewöhnlich gegen Ende des zweiten Wettbewerbsjahres abgehalten wird.

5. Die für die Teilnahme am Wettbewerb bestimmten Arbeiten sind in sechsfacher Ausfertigung an den Deutschen Sportbund, Abteilung Bildung und Wissenschaft, OttoFleck-Schneise 12, 60528 Frankfurt am Main, zu senden.

Einsendeschluß ist der 31. März 2000 (Poststempel).

6. Den eingereichten Arbeiten sind folgende Angaben beizufügen:

a) Name, Anschrift und kurzgefaßter Lebenslauf des Verfassers oder der Verfasserin;

b) eine eidesstaatliche Erklärung, daß die Arbeit selbständig angefertigt wurde und daß die Bewerbung den Bedingungen der Ausschreibung gerecht wird;

c) eine vollständige Zusammenstellung der benutzen Hilfsmittel und die Versicherung, daß keine anderen Hilfsmittel benutzt wurden (soweit diese Angaben nicht bereits in der Arbeit selbst enthalten sind);

d) eine Erklärung, ob, wo und in welcher Fassung die Arbeit bereits Gegenstand eines Wettbewerbs war oder ist.

7. Die Arbeiten dürfen bis zum Abschluß des Wettbewerbs (mit der Festakademie) in der eingereichten Form noch nicht veröffentlicht sein. Jeweils eine davon geht als Belegstück in das Eigentum des DEUTSCHEN SPORTBUNDES über.

8. Mit ihrer Teilnahme am Wettbewerb übertragen die Bewerber und Bewerberinnen dem Deutschen SPORTBund das Recht, eine mit der Plakette oder einem anderen Preis ausgezeichnete Arbeit in der "Wissenschaftlichen Schriftenreihe des Deutschen Sportbundes" zu veröffentlichen. Die Veröffentlichungen werden in der Regel bezuschußt.

Frankfurt am Main, im September 1999

Kuratorium für die Verleihung der Carl-Diem-Plakette gez. Professor Dr. Ommo Grupe Vorsitzender 